\title{
MARE NOSTRUM
}

StUdENTISCHE BEITRÄGE ZUR MEDITERRANISTIK

Band 1 (2021)

Reiseerfahrungen im Mittelmeerraum in Mittelalter und Moderne

Herausgegeben von Margit Mersch 


\title{
Mare Nostrum. Studentische Beiträge ZUR MediterRanistik
}

\author{
Herausgeberin \\ Margit Mersch (Bochum)

\section{REDAKTIONSANSCHRIFT} \\ Dr. Margit Mersch \\ Historisches Seminar, Geschichte des Mittelalters \\ Ruhr-Universität Bochum \\ GA 4/33 Fachnr. 182 \\ Universitätsstraße 150 \\ 44801 Bochum \\ Margaretha.Mersch@rub.de
}

\section{Website}

https://ojs.ub.rub.de/index.php/MaNo

Alle Informationen zur Zeitschrift, wie etwa Hinweise für Autoren etc. sind auf der Website zu finden. Wenn Sie Interesse an der Veröffentlichung eines Beitrags oder an der Herausgabe eines Themenheftes haben, wenden Sie sich bitte an die Herausgeberin.

\section{ISSN 2749-3091}

\section{KONZEPT}

MARE NOSTRUM ist ein interdisziplinäres open-access e-Journal, das Beiträge von Nachwuchswissenschaftlerinnen und -wissenschaftlern aus allen Bereichen der wissenschaftlichen Mediterranistik veröffentlicht. Es fungiert insbesondere als ein Publikationsorgan für studentische Arbeiten zum Themenbereich "Mittelmeer", die aus thematisch einschlägigen Lehrveranstaltungen der Ruhr-Universität Bochum hervorgegangen sind. Darüber hinaus bietet die Zeitschrift allen interessierten Studierenden sowie Wissenschaftlerinnen und Wissenschaftlern eine Plattform zur Vorstellung ihrer Forschungsvorhaben oder -ergebnisse, die einen Bezug zum Mittelmeerraum aufweisen. MARE NOSTRUM wird unter dem Dach des Zentrums für Mittelmeerstudien an der Ruhr-Universität Bochum herausgegeben und gehostet von der Universitätsbibliothek der Ruhr-Universität Bochum.

Umschlag-Abbildung: Richard William Seale: A correct Chart of the Mediterranean Sea, from the Straits of Gibraltar to the Levant. From the latest and best Observations: for Mr. Tindal's Continuation of Mr. Rapin's History, London 1745. Wikimedia Commons, File:1745 Seale Map or Chart of the Mediterranean Sea - Geographicus - Mediterraneanseale-1845.jpg (09.06.2021). 


\section{Inhaltsverzeichnis}

\section{Margit Mersch}

Editorial: Reiseerfahrungen im Mittelmeerraum

\section{Mike Erdtmann}

Maritimes Wissen. Hierarchie und Alltag auf venezianischen Pilgergaleeren des ausgehenden 15. Jahrhunderts

Florian Müller

Der Blick als Erfahrung? Fremdbeschreibung und Selbstbeschreibung als erzählende Wissensformen am Beispiel des Reiseberichts des Bernhard von Breydenbach

Jan-Niklas Graf

Der Pilger und die Fremden. Ibn Dschubair über christlich-muslimische

Koexistenz

Zoe Mießner

Frömmigkeit und Wissensdurst. Der Reisebericht Arnolds von Harff

Cedric Lorra

Ein muslimischer Reisender unter Christen. Ibn Dschubairs Erfahrungen im Heiligen Land und im normannischen Sizilien.

Till Peters

Piraterie im spätmittelalterlichen Mittelmeerraum. Akteure, Gründe und Motive und die Angst christlicher Pilger vor muslimischen Piraten

Rouven Thäwel

Nautisch-militärische Technologie beim vierten Kreuzzug 1202-1204

Anna Breidenbach

Die Erfahrung der Nation. ,Modernes Reisen“ auf der "Augusta Victoria« (1891) 